\title{
Coronavirus Disease 2019 in a Hemodialysis Patient
}

\author{
Hai Yuan ${ }^{\mathrm{a}}$ E. Guo ${ }^{\mathrm{b}}$ Zhao Gao ${ }^{\mathrm{a}}$ Fengqi Hu ${ }^{\mathrm{a}}$ Li Lu ${ }^{\mathrm{a}}$ \\ ${ }^{a}$ Department of Nephrology, Xiangyang Central Hospital, Affiliated Hospital of Hubei University of Arts and Science, \\ Xiangyang, China; ${ }^{b}$ Department of Respirology, Xiangyang Central Hospital, Affiliated Hospital of Hubei University \\ of Arts and Science, Xiangyang, China
}

\section{Keywords}

Coronavirus disease $19 \cdot$ Severe acute respiratory

syndrome-CoV-2 · Hemodialysis · End-stage renal disease

\begin{abstract}
There has been a global outbreak of the coronavirus disease 2019 (COVID-19) since December 2019. Here, we describe the case of a 49-year-old male undergoing maintenance hemodialysis (HD) who got infected with COVID-19 and our experience in performing HD for him. The patient's symptoms and lung imaging changes were atypical. However, his lymphocyte range decreased upon admission and the polymerase chain reaction of the pharyngeal swab for the COVID-19 nucleic acid was positive. The patient developed respiratory failure and required mechanical ventilation 8 days after admission. In the end, he died from multiple organ dysfunction syndrome. The difficulties in diagnosis, infection control, and treatment of COVID-19 in maintenance HD patients are discussed in this report. (0) 2020 S. Karger AG, Basel
\end{abstract}

\section{Introduction}

Since early December 2019, an outbreak of the coronavirus disease 2019 (COVID-19) occurred in Wuhan, China. The virus was identified as belonging to the genus Betacoronavirus, placing it alongside severe acute respi- ratory syndrome (SARS) and Middle East respiratory syndrome (MERS) viruses [1]. It has affected $>100,000$ people in over 50 countries, including China, Korea, Italy, Iran, Japan, France, Germany, and the United States [2]. The Hubei province of China has been one of the most severely affected areas; $>65,000$ people contracted COVID-19 [2].

Patients with end-stage renal disease and undergoing maintenance hemodialysis (HD) are at greater risk of viral transmission due to impaired immune function and high co-morbidity [3-5]. Moreover, they are exposed to frequent and repetitive potentially infectious risk factors during regular HD treatment [6]. Previous studies found that HD facilities had developed into one of the principal clusters for SARS and MERS infections [7, 8].

To date, there have been relatively few cases of COVID-19 patients requiring maintenance HD. Here, we describe our experience in the management of a maintenance HD patient who developed COVID-19 in Xiangyang, China.

\section{Case Report}

A 49-year-old male had end-stage renal disease and secondary to diabetic nephropathy, and was undergoing maintenance HD. His general condition was stable. He was admitted to the hospital

H.Y. and E.G. contributed equally to this work.
KARGER

(C) 2020 S. Karger AG, Basel

E-Mail karger@karger.com

www.karger.com/bpu
Fengqi $\mathrm{Hu}$ and $\mathrm{Li} \mathrm{Lu}$

Department of Nephrology, Xiangyang Central Hospital

Affiliated Hospital of Hubei University of Arts and Science

Xiangyang, Hubei 441000 (China)

E-Mail hufengqi1@gmail.com and 1549031579@ qq.com 

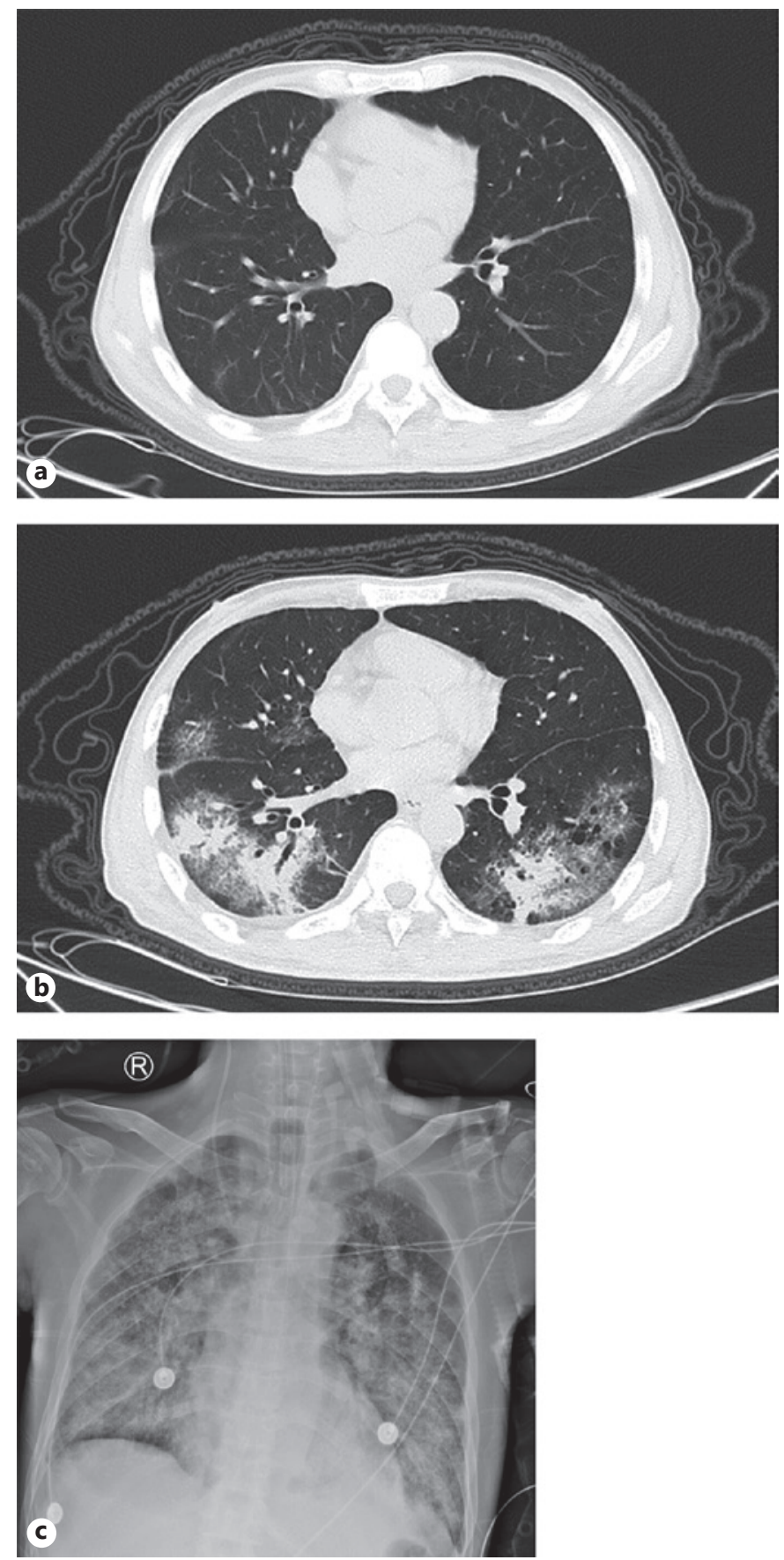

Fig. 1. Radiographic images of the patient. a An axial CT of the thorax on admission showing a small cotton-wool spot in the right lobe of the right lung. $\mathbf{b}$ An axial CT of the thorax recorded 8 days after admission showing diffuse large regions of ground-glass opacification with partial consolidation in the lower lobe of both lungs. c Chest radiograph 14 days after admission showing several reticular shadows in both the lung fields, especially in bilateral middle and lower lobes. on January 31,2020 , with complaints of intermittent cough. However, he had no fever, chills, myalgia, or anorexia before admission. He had undergone chest computed tomography (CT) scan because of a history of contact with a COVID-19 patient (his mother) 5 days earlier. His CT scan showed a small cotton-wool spot in the right lobe of the right lung and he was suspected to have COVID-19 (Fig. 1a). The reverse transcription polymerase chain reaction (RT-PCR) of the patient's pharyngeal swab was positive for the COVID-19 nucleic acid 2 days after hospitalization. The patient was treated with lopinavir plus ritonavir combined with interferon alfa-2b inhalation as antiviral therapy, and continued on HD (see below). However, 5 days after admission, he began to develop fever and his condition worsened rapidly. A repeat chest CT showed scattered ground-glass opacities in both lungs 8 days after admission (Fig. 1b). He was transferred to the intensive care unit and required endotracheal intubation and mechanical ventilation. At 14 days after admission, chest radiography showed several reticular shadows in both lung fields (Fig. 1c). Unfortunately, after 15 days of admission, the patient developed multiple organ dysfunction syndrome and died.

Precautionary measures were applied in our HD center during the COVID-19 outbreak in late December 2019, a month before this patient was confirmed to have COVID-19. These included enhanced environmental cleaning, exclusion of visitors, and monitoring of hand hygiene. All patients undergoing HD had their body temperatures monitored and were asked to put on masks; patients with fever had to undergo chest CT and blood cell analysis. This allowed shifting of suspected patients into an isolated dialysis unit in isolated wards. Since an outbreak of the COVID-19 developed in Hubei, China, especially after presence of the first COVID-19 positive patient in our HD unit, some maintenance HD outpatients worried about becoming infected and prolonged their HD interval. The dialysis unit staff followed the recommendations of the WHO [9] for infection control including the use of waterproof disposable gowns, gloves, caps, goggles, and N95 masks.

Once the diagnosis of COVID-19 was confirmed, the patient was kept in the COVID-19 isolation ward along with other nondialysis COVID-19 patients; the dialysis machine was kept in the COVID-19 isolation ward between dialysis sessions and was used only for COVID-19 patients requiring HD. All staff involved in the direct care of patients affected by COVID-19 had to undertake full protection (see above) and strictly implemented hand hygiene. We performed continuous venovenous hemodiafiltration using the Fresenius multiFiltrate dialysis machine (Fresenius Medical Care, Bad Homburg, Germany). A commercial carbonate replacement fluid (QingShanLiKang, Chengdu, China) was used and Fresenius AV600S dialyzer (Fresenius Medical Care) was employed without reuse. After each HD session, the dialyzer, all blood tubing, and the spent dialysate were discarded as infectious waste and the dialysis machine was disinfected with sodium hypochlorite solution according to the manufacturer's instructions.

\section{Discussion/Conclusion}

Since COVID-19 is highly contagious by close contact, infection control in the HD units is of utmost importance for nephrologists practicing in epidemic areas. There are 
many challenges in the management of maintenance dialysis patients who contract COVID-19, such as difficulty in early diagnosis, adjustments required in deciding the dose of antiviral medications, additional infection-control issues in the disposal of the spent dialysate, and prevention of cross-contamination within the dialysis unit.

Since the beginning of the outbreak of COVID-19, all HD patients and staff of the HD unit were tested for COVID-19 according to their history of close contact with COVID-19 patients and symptom. All HD inpatients and outpatients and staff of an HD unit who have fever, new cough, or dyspnea should be screened for COVID-19 infection by blood cell analysis, chest CT, and RT-PCR of their pharyngeal swab (if available, since the nucleic acid detection resources were limited during January in China). Since the Hubei province in China was one of the most severely affected areas, based on the recommendations of the Hubei COVID-19 Epidemic Prevention and Control Command in February 2020, all patients on HD and staff of the HD unit were tested with blood cell analysis, chest CT, and RT-PCR of their pharyngeal swab. No further cases of secondary transmission were detected among the HD patients and the staff in our dialysis unit. After that, all new inpatient HD patients were required to be screened for COVID-19 infection.

In this report, we describe the clinical course of a maintenance HD patient who developed COVID-19, our experience in providing $\mathrm{HD}$ for him, and the precautionary measures that were applied in our dialysis unit. The initial clinical presentation of our patient was completely asymptomatic, except for intermittent coughing and sputum at presentation. A recent study of 1,099 patients with laboratory-confirmed COVID-19 indicated that fever was identified only in $43.8 \%$ patients on admission and the absence of fever in COVID-19 infections was more frequent than in SARS and MERS [1]. Previous studies found that HD patients tend to have less pronounced symptoms of SARS [10-12], which may be difficult to distinguish from uremic symptoms. Therefore, epidemiological investigation of HD patients and the detection of symptoms and timely tests are particularly important for identifying a potential COVID-19 infection in HD patients.

Appropriate infection-control precautions such as waterproof disposable gowns, gloves, caps, face shields or goggles, and N95 masks are useful for protection against SARS and MERS in the HD units [7, 13]. A recent study showed that $3.5 \%$ of COVID-19 infected patients were healthcare workers [14]. We worked closely with the Infection Control Unit of the hospital to enforce proper infection-control measures, and no staff or other patients in the HD unit were infected. The medical staff in the HD units were in contact with potential COVID-19 infected patients. The entire staff of the HD unit were required to monitor themselves for COVID-19 and the clinical staff were reminded to look out for suspected COVID-19 cases to facilitate early identification and isolation. Infection-control precautions were used to protect against COVID-19 infection. Since the COVID 19 virus is approximately $60-140 \mathrm{~nm}$ in diameter [15], it should not pass through the conventional low-flux polysulfone membrane like SARS-CoV does [12]. Once the diagnosis of COVID-19 was confirmed, disinfection was carried out immediately. Areas in close contact with the confirmed patient were not used for other patients until these areas were completely cleared. Screening of other HD patients in the same section of the unit and/or on the same shift with the confirmed patient and staff who took care of the confirmed patient was necessary to allow for early recognition and isolation.

In summary, we described anHD patient with COVID-19 infection. The experiences in our case show that COVID-19 in maintenance patients may have an atypical presentation with minimal symptoms, leading to diagnostic difficulties. Detecting COVID-19 infection at an earlier stage may result in immediate isolation and decrease in the spread of COVID-19. Recently, several recommendations about prevention and control of the emerging COVID-19 pandemic in HD centers were published [16-18], providing more information for management of $\mathrm{HD}$ centers during the COVID-19 outbreak. With appropriate infection-control measures, like wearing protective gear, the staff taking care of the concerned patient and other maintenance HD patients were protected from infection.

\section{Acknowledgment}

We would like to thank Editage (www.editage.cn) for English language editing.

\section{Statement of Ethics}

Written informed consent was obtained from the patient's mother, including consent for data collection and written informed consent for publication of the details of the case including images.

\section{Disclosure Statement}

The authors have no conflicts of interest to declare. 


\section{Funding Sources}

This work was funded by grants from the National Natural Science Foundation of China (No. 81300617) and the Natural Science Foundation of Hubei Province (No. 2019CFB597).

\section{Author Contributions}

H.Y. conceived the case report and collected the data. H.Y. and Z.G. wrote the manuscript. F.H. was involved in the supervision of the work and the critical revision of the paper.

\section{References}

1 Peeri NC, Shrestha N, Rahman MS, Zaki R, Tan Z, Bibi S, et al. The SARS, MERS and novel coronavirus (COVID-19) epidemics, the newest and biggest global health threats: what lessons have we learned? Int J Epidemiol. 2020, Epub ahead of print.

2 World Health Organization. Coronavirus disease 2019 (COVID-19) Situation Report - 41. https://www.who.int/docs/default-source/ coronaviruse/situation-reports/20200301-sitrep-41-covid-19.pdf?sfvrsn=6768306d_2.

3 Dalrymple LS, Go AS. Epidemiology of acute infections among patients with chronic kidney disease. Clin J Am Soc Nephrol. 2008 Sep; 3(5):1487-93.

4 Kato S, Chmielewski M, Honda H, PecoitsFilho R, Matsuo S, Yuzawa Y, et al. Aspects of immune dysfunction in end-stage renal disease. Clin J Am Soc Nephrol. 2008 Sep;3(5): 1526-33.

5 Butler AM, Layton JB, Dharnidharka VR, Sahrmann JM, Seamans MJ, Weber DJ, et al. Comparative Effectiveness of High-Dose Versus Standard-Dose Influenza Vaccine Among Patients Receiving Maintenance Hemodialysis. Am J Kidney Dis. 2020 Jan;75(1):72-83.

6 Sarnak MJ, Jaber BL. Mortality caused by sepsis in patients with end-stage renal disease compared with the general population. Kidney Int. 2000 Oct;58(4):1758-64.
7 Wong PN, Mak SK, Lo KY, Tong GM, Wong $\mathrm{Y}$, Watt CL, et al. Clinical presentation and outcome of severe acute respiratory syndrome in dialysis patients. Am J Kidney Dis. 2003 Nov;42(5):1075-81.

8 Assiri A, McGeer A, Perl TM, Price CS, Al Rabeeah AA, Cummings DA, et al.; KSA MERS-CoV Investigation Team. Hospital outbreak of Middle East respiratory syndrome coronavirus. N Engl J Med. 2013 Aug; 369(5):407-16.

9 World Health Organization. https://www. who.int/emergencies/diseases/novel-coronavirus-2019/technical-guidance/infectionprevention-and-control.

10 Kwan BC, Leung CB, Szeto CC, Wang AY, Li PK. Severe acute respiratory syndrome in a hemodialysis patient. Am J Kidney Dis. 2003 Nov;42(5):1069-74.

11 Tang HL, Cheuk A, Chu KH, Lee W, Wong $\mathrm{SH}$, Cheng YL, et al. Severe acute respiratory syndrome in haemodialysis patients: a report of two cases. Nephrol Dial Transplant. 2003 Oct;18(10):2178-81.

12 Kwan BC, Leung CB, Szeto CC, Wong VW, Cheng YL, Yu AW, et al. Severe acute respiratory syndrome in dialysis patients. J Am Soc Nephrol. 2004 Jul;15(7):1883-8.
13 Park HC, Lee YK, Lee SH, Yoo KD, Jeon HJ, Ryu DR, et al.; Korean Society of Nephrology MERS-CoV Task Force Team. Middle East respiratory syndrome clinical practice guideline for hemodialysis facilities. Kidney Res Clin Pract. 2017 Jun;36(2):111-6.

14 Guan WJ, Ni ZY, Hu Y, Liang WH, Ou CQ, He JX, et al.; China Medical Treatment Expert Group for Covid-19. Clinical Characteristics of Coronavirus Disease 2019 in China. N Engl J Med. 2020, Epub ahead of print.

15 Zhu N, Zhang D, Wang W, Li X, Yang B, Song J, et al.; China Novel Coronavirus Investigating and Research Team. A Novel Coronavirus from Patients with Pneumonia in China, 2019. N Engl J Med. 2020 Feb;382(8):727-33.

16 Basile C, Combe C, Pizzarelli F, Covic A, Davenport A, Kanbay M, et al. Recommendations for the prevention, mitigation and containment of the emerging SARS-CoV-2 (COVID-19) pandemic in haemodialysis centres. Nephrol Dial Transplant. 2020, Epub ahead of print.

17 Ikizler TA. COVID-19 and Dialysis Units: What Do We Know Now and What Should We Do? Am J Kidney Dis. 2020, Epub ahead of print.

18 Kliger AS, Silberzweig J. Mitigating Risk of COVID-19 in Dialysis Facilities. Clin J Am Soc Nephrol. 2020, Epub ahead of print. 